\title{
"Je suis la voix de la pratique»
}

\section{Daniel Lüthi}

Journaliste indépendant et photographe, conseiller médiatique, Berne

L'image est tout un symbole: une infirmière - peut-être encore une élève - traverse d'un pas sûr et décidé la pelouse devant l'école. Pia Coppex porte au monument un regard presque admiratif: «Oui», dit l'enseignante, «nous pouvons être fières de notre métier.» La sculpture de Denis Perret-Gentil lui plaît surtout pour le message qu'elle véhicule.

\section{L'infirmière devenue présidente}

A l'Institut et Haute Ecole de la Santé «La Source» à Lausanne, Pia Coppex est non seulement enseignante, mais aussi responsable du plan d'études DAS «Santé des populations vieillissantes» depuis presque une année. Au niveau national, c'est une autre fonction qui l'a fait connaître du public: elle est en effet la première infirmière à être devenue présidente de la Société Suisse d'Ethique Biomédicale (SSEB). Elue en avril à la tête de cette organisation de plus de 20 membres, elle se retrouve entourée de nombreux porteurs de titres académiques: Prof. Dr med., Prof. Dr théol., Dr phil., dipl. psych., Dr iur., etc.

Pia Coppex n'a pas de doctorat, mais une solide formation et beaucoup d'expérience. Elle insiste sur le fait qu'elle est toujours restée proche de la base, des gens «du terrain» et de leurs préoccupations dans sa car-

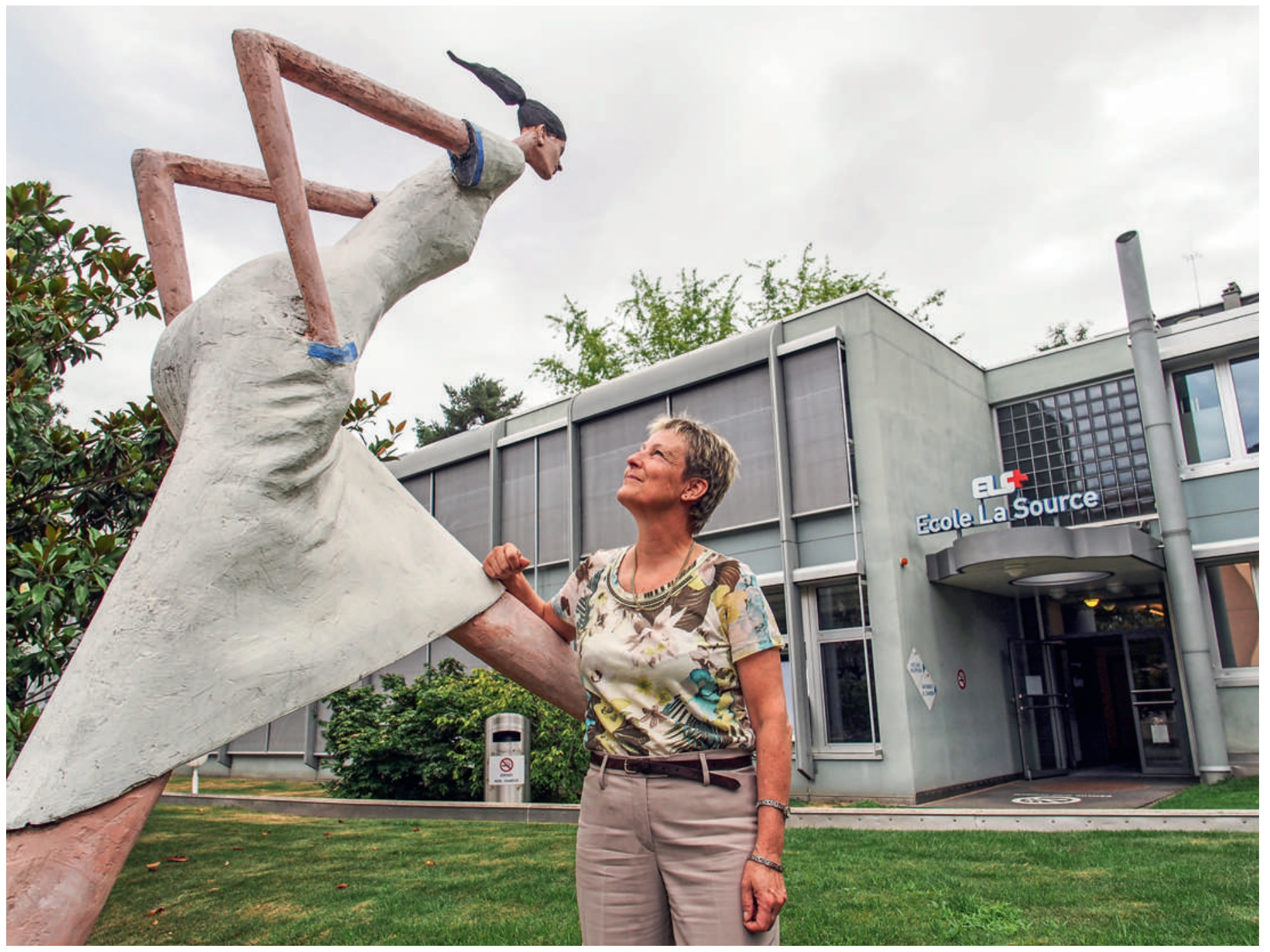


rière professionnelle. "Je suis la voix de la pratique», dit-elle, «et j'ai été très honorée que l'on ait pensé à moi pour cette élection.» Pourquoi aspirait-elle à cette fonction qui implique davantage de responsabilités? "Le système de santé est devenu complexe et il est important pour moi de réunir les différents acteurs. De plus, à une époque où les arguments économiques ont toujours plus de poids, il est important que l'on prenne en compte d'autres valeurs.» La responsabilité, les droits humains, la dignité et le respect, voilà les valeurs qu'elle défend quand elle parle d'éthique. Des valeurs qu'elle a toujours défendues comme prioritaires, y compris au lit du malade.

\section{Où est la bonne solution?}

Pia Coppex cite un exemple: une femme qui vivait chez elle, mais souffrait d'une démence progressive. Elle était de moins en moins capable de prendre soin d'elle-même et ne cuisinait presque plus. Elle a fait une chute et s'est blessée en s'ébouillantant avec du café. «Sa nièce a donné l’alerte, mais la femme voulait rester chez elle.» Une décision s'imposait, qui devait être prise d'entente avec toutes les personnes impliquées: le médecin de famille, le personnel infirmier, la nièce - et la femme concernée. «Dans un tel cas, pas question de se limiter à des aspects formalistes, ni surtout de laisser tomber une personne et ses proches." Qu'est-ce qui donne du sens à ce que vit cette personne en ce moment-même? Quel est le bien véritable? Toute la question est là.

\section{«Il s'agit d'opérer une synthèse entre différents angles de vision.»}

Prenons un deuxième exemple: une femme avait besoin de séances de physiothérapie pour réapprendre à marcher convenablement, renforcer ses muscles, en un mot s'exercer. Une collègue de Pia Coppex a assis la dame dans un fauteuil roulant pour l'amener à sa séance de physiothérapie. "C'est un non-sens total», s'emporte Coppex, «ma collègue ici n'a tout simplement pas réfléchi! Il ne suffit pas de dire 'je fais ça pour ton bien'. La bonne solution se trouve parfois dans des comportements évidents dans la vie quotidienne.»

\section{L'importance du travail d'équipe}

Bien sûr, il n'est pas toujours facile de trouver la manière idéale; les acteurs du système de santé sont régulièrement confrontés à des situations humainement difficiles qui n'ont pas de solutions toutes faites. C'est

\section{Pia Coppex-Gasche}

Pia Coppex-Gasche est née en 1961 à Soleure. A l'âge de six ans, elle a déménagé avec sa famille à Monthey, dans le Bas-Valais, où elle a fait ses écoles et suivi sa formation d'infirmière en psychiatrie. Diplômée en 1983, elle a ensuite travaillé pendant trois ans au service des urgences de

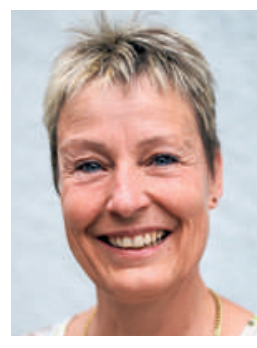

I'hôpital psychiatrique de Monthey, avant de suivre plusieurs formations postgrades, notamment en gérontologie, en psychologie du vieillissement, en droit de la santé, en pédagogie et en éthique. De 2011 à 2014, elle a été cheffe de projet à la Conférence des directeurs de la santé CDS à Berne. Depuis septembre 2014, elle est enseignante et responsable du plan d'études "Santé des populations vieillissantes" à la Haute Ecole Spécialisée "La Source» à Lausanne. En avril 2015, elle a été la première infirmière élue présidente de la Société Suisse d'Ethique Biomédicale. Pia Coppex est mariée et mère de deux filles adultes. Elle vit avec son époux à Choëx-sur-Monthey.

ce qui rend le travail d'équipe si essentiel: «Une équipe qui a appris à travailler ensemble est en meilleure position pour prendre la décision suivante.» Elle-même n'a jamais buté sur des obstacles hiérarchiques entre les corps infirmier et médical, ni ressenti des attitudes suffisantes de la part des médecins. «En psychiatrie, la collaboration interdisciplinaire était quelque chose qui allait de soi, je l'ai su dès ma formation.» C'est ce qui explique qu'elle ne regarde pas les médecins comme des dieux, «nous sommes collègues». Comme le sont tous les autres qui prennent à cœur le bien-être des patients. «J'ai toujours fait de belles rencontres et beaucoup sont devenus des amis", raconte Pia Coppex. La nouvelle présidente de la SSEB a prévu de prendre le temps de rencontrer et faire plus ample connaissance de chaque membre du comité, fidèle en cela à la devise de la société. Celle-ci a été fondée en 1989, écrit-elle, dans le but de chercher «la concordance par delà les frontières des différentes disciplines», ce qui, de toute évidence, ne va pas de soi.

«Il s'agit d'opérer une synthèse entre différents angles de vision, de créer le dialogue - dans le but de se comprendre et de se mettre d'accord, donc de trouver un consensus.» Un compromis n'est pas toujours possible ni nécessaire, explique Pia Coppex, qui illustre ses déclarations abstraites par un exemple concret. La question était: devions-nous approuver ou non un avortement chez une jeune femme? «Mon avis initial était à l'opposé de la décision finalement prise. Mais je m'y suis ralliée parce que nous l'avions prise en équipe dans une attitude solidaire.» 


\section{La déferlante grise...}

Pia Coppex est spécialisée dans les problèmes de lâge, dont la démence et les soins palliatifs. Des thèmes qui, soit dit en passant, nous rappellent en permanence nos propres limites et la fugacité de notre existence. Pourquoi a-t-elle fait précisément ce choix dans sa jeunesse? «Notre démographie est ce qu'elle est; la 'déferlante grise' est une réalité et un défi", répond-elle après un instant de réflexion. «Nous devons apprendre à utiliser très efficacement nos ressources si nous voulons que notre système de santé réponde encore aux besoins de la population dans les années à venir.» Comme presque toujours dans un moment pareil, elle

\section{"Je serais heureuse de travailler pour l'Organi- sation Mondiale de la Santé (OMS).»}

porte alors au loin un regard éloquent, cherche ses pensées et choisit spontanément de les formuler en suisse-allemand ou en français. Puis elle respire profondément et se tourne en souriant vers son interlocuteur: «Les personnes âgées m’ont toujours énormément apporté», dit-elle avec passion. «Même celles qui sont démentes. Elles n'ont peut-être plus de mots mais ces regards! Avec notre regard et notre façon de leur parler, nous pouvons faire en sorte qu'elles restent des personnes à part entière, des êtres humains avec une histoire, une destinée et une exigence d'autonomie - et qu'elles ne deviennent pas des objets.» On retrouve ici le concept de la dignité humaine, et avec lui la thématique souvent si abstraite et insaisissable de l'éthique.

\section{... et le problème du temps}

Avoir le temps, se prendre le temps d'écouter - cela devrait faire partie de notre métier. Cela devrait même en être un élément central. Seulement voilà: toutes celles et tous ceux qui travaillent dans le domaine de la santé se plaignent de manquer de temps. Pia Coppex: «Dès lors que la performance et les exigences administratives sont en jeu, les infirmiers et infirmières et les médecins sont souvent amenés à trahir leurs propres valeurs et convictions - avec le risque de se perdre.» Elle ajoute cependant: «Le problème du temps est relatif. Le temps n'est pas une question de quantité mais de qualité, de présence et de disponibilité. Il suffit parfois d'un regard, de quelques paroles succinctes mais sincères.»
Ce qui compte pour l'infirmier comme pour le médecin, c'est de ne pas s'effacer mais de rester soi-même. Il est légitime de se défendre, dit Coppex, mais cela ne fait nullement d'elle une révolutionnaire. Elle reste aimable et joyeuse tout en affichant sa détermination. Et elle dit: «Le patient est au centre de notre action. Il appartient à tous les membres d'une équipe interprofessionnelle de s'en occuper le mieux possible. Pour cela, il faut le dialogue.»

\section{Le Valais et le monde}

Dans le petit bureau qu'elle partage, une affiche promotionnelle pour le Valais figure bien en vue sur la paroi. Elle le dit et le répète, le Valais est le lieu de son cœur. Elle y est chez elle et fait la navette pour se rendre à Berne et à Lausanne. Sa famille, ses amis, le jardin et la montagne comptent beaucoup pour elle, "tout comme le fait qu'en Valais, on ose se dire les choses franchement». Son ancrage professionnel et géographique y est très fort.

Dans ses loisirs, elle compose des vitraux en assemblant des morceaux de verre coloré avec du plomb. La multiplicité des couleurs a valeur de symbole pour cette femme, y compris dans sa vie professionnelle. Elle passe son temps à réunir des gens de différents métiers; en ce moment, elle est en train de mettre sur pied un congrès intercantonal à Lausanne sur un thème qui lui est cher, «Vieillir en Suisse». Pia Coppex est femme de réseaux et voyage beaucoup à partir de ses bases, $y$ compris en pensées.

Elle n'est donc pas du genre à tenir en place. Elle veut aller toujours plus loin, avancer fièrement comme la sculpture de l'école «La Source» à Lausanne.

Quel est son prochain objectif? Coppex rit: «J'ai travaillé dans plusieurs institutions cantonales et nationales. Maintenant, je me verrais bien entrer dans une structure politique internationale. S'il m'est permis de rêver un peu, je serais heureuse de travailler pour l'Organisation mondiale de la Santé (OMS).»

Soyons certains que même là-bas, elle ne voudrait pas perdre le contact avec sa base, avec la pratique du métier.

\footnotetext{
La prochaine «Rencontre avec...»

A la fin de chaque mois, le Bulletin des médecins suisses pré sente une personnalité qui s'implique dans la santé publique. En septembre, Daniel Lüthi parlera de sa rencontre avec Daniel Hell, professeur émerité en psychiatrie.
} 